\title{
Stromal Cell-Derived CCL20 Promotes Tumor Progression and Osteolysis in Giant Cell Tumor of Bone
}

\author{
Chenglong Zhao Dongsheng Wang ${ }^{\mathrm{a}, \mathrm{b}}$ Liang Tang ${ }^{\mathrm{a}, \mathrm{b}} \quad$ Zhichao Zhang ${ }^{\mathrm{a}}$ Song $\mathrm{Li}^{\mathrm{a}}$ \\ Ming Qian ${ }^{\mathrm{a}}$ Zhipeng Wu $\mathrm{W}^{\mathrm{a}}$ Wang Zhou ${ }^{\mathrm{a}}$ Mingyao Liu ${ }^{\mathrm{b}, \mathrm{c}}$ \\ Jian Luo ${ }^{b, c}$ Tielong Liua ${ }^{a, b}$ Zhenxi Lia,b Jianru Xiao ${ }^{a, b}$ \\ aSpine Tumor Center, Department of Orthopedic Oncology, Changzheng Hospital, Second Military \\ Medical University, Shanghai, 'East China Normal University and Shanghai Changzheng Hospital Joint \\ Research Center for Orthopedic Oncology, Shanghai, 'Shanghai Key Laboratory of Regulatory Biology, \\ Institute of Biomedical Sciences and School of Life Sciences, East China Normal University, Shanghai, \\ China
}

\section{Key Words}

$\mathrm{CCL} 20 \cdot$ Giant cell tumor of bone $\cdot$ Cell viability $\bullet$ Cell migration $\bullet$ Osteoclastogenesis

\begin{abstract}
Background/Aims: Giant cell tumor of bone (GCTB), one of the most common primary bone tumors, leads to extensive bone destruction. However, the mechanisms underlying GCTB progression remain elusive and prognostic factors and treatment targets are required. In the current study, we explored the function of the chemokine family member CCL20 in GCTB progression. Methods: We explored the expression of CCL20 in stromal cells (GCTSCs) using microarray. Clinical analyses of the role of CCL20 in tumor progression were performed based on the patient cohort of our institution. The role of CCL20 in tumor proliferation was evaluated by MTS assay, migration ability was measured by a Transwell assay, and osteoclastogenesis was induced by CCL20 or GCTSC-conditioned medium. Quantitative PCR and western blot were used to measure the expression levels of mRNAs and proteins related to tumor progression. Results: CCL20 was upregulated in GCTSCs and correlated with tumor progression and prognosis. CCL20 induced GCTSC proliferation and migration in an autocrine manner. In addition, CCL20 recruited mononuclear cells and induced osteoclastogenesis by overactivating the AKT and NF-KB signaling pathways. Antibody blockade of CCL20 abolished the exacerbated osteoclastogenesis. Conclusion: Taken together, our data indicate that GCTSC secretion of CCL20 acts as a key modulator in the pathological progression of GCTB. It can promote GCTSC proliferation and migration in an autocrine manner and can recruit
\end{abstract}


bone marrow monocytes to the tumor microenvironment and enhance osteoclastogenesis in a paracrine manner. These findings strongly indicate the potential prognostic and therapeutic value of CCL20 in GCTB.

\section{Introduction}

Giant cell tumor of bone (GCTB) is one of the most common primary bone tumors, accounting for approximately $5 \%$ of all bone tumors [1,2]. GCTB predominantly occurs in the metaphysis, thoracic vertebra, and sacrum of individuals in their third and fourth decades of life and shows a female predilection [1,3-5]. This kind of tumor is osteolytic and comprises three major cell populations: osteoclast-like multinuclear giant cells, mononuclear precursors of osteoclasts, and spindle-shaped stromal cells (GCTSCs) [6-8]. GCTSCs are considered to be the tumor component, but the exact function of GCTSCs in the tumor microenvironment remains elusive.

GCTB is classified as a benign but local aggressive tumor with a 1-9\% incidence of lung metastasis [9]. Currently, the most effective treatment for GCTB is surgical excision. En bloc resections are recommended but not always viable due to tumor size or local anatomical structures. As a result, insufficient surgical margins lead to high local recurrence rates [1, 5]. Bisphosphonates and denosumab can be used to control bone destruction but have little effect on GCTSCs [8]. Thus, proper prognostic markers and therapeutic targets are urgently needed for GCTB.

Chemokines are small chemo-attractant proteins distributed among four subfamilies named C, CC, CXC, and CX3C $[10,11]$. Members of the chemokine family biologically interact with chemokine receptors, which are all G-protein coupled receptors, and act as mediators in the processes of cell proliferation, migration, and differentiation [12,13]. Previous studies have indicated that chemokines play important roles in tumor pathology and progression [14, 15]. CC-chemokine ligand 20 (CCL20), also known as macrophage inflammatory protein-3 alpha (MIP-3 $\alpha$ ), is expressed mainly in mononuclear cells, T lymphocytes, and endothelial cells. CCL20 can be induced by a variety of cytokines, such as tumor necrosis factor alpha (TNF $\alpha$ ) and interleukin 1 (IL-1) [16-18]. CCL20 is upregulated in some solid tumors, such as breast, liver, colon, and pancreatic tumors. Furthermore, CCL20 is associated with tumor progression and prognosis [19-23]. However, the functions of CCL20 in GCTB have not been examined.

In the current study, we found that CCL20 was upregulated in GCTSCs compared with normal bone marrow stromal cells (BMSCs). The serum CCL20 level was also elevated, which correlated with tumor progression and patient outcomes. We further confirmed that CCL20 can promote GCTSC proliferation and migration in an autocrine manner while recruiting and inducing mononuclear cells to differentiate into osteoclasts. Antibody blockade of CCL20 abolished the exacerbated osteoclastogenesis. These results indicate the potential value of CCL20 in GCTB progression and show that CCL20 might be a promising prognostic factor and therapeutic target in GCTB patients.

\section{Materials and Methods}

\section{Patients and microarray analysis}

A total of 32 GCTB patients and 13 normal donors were involved in this study. Tumor samples, bone marrow cells, and peripheral blood samples were collected after the participants provided written informed consent. The study was approved by the Ethics Committee of Changzheng Hospital. Total mRNA was extracted from five GCTSCs and five BMSCs and subjected to microarray analysis. The results were further analyzed to elucidate the changes in the expression levels of chemokine family members. 


\section{Cellular Physiology Cell Physiol Biochem 2018;51:2472-2483

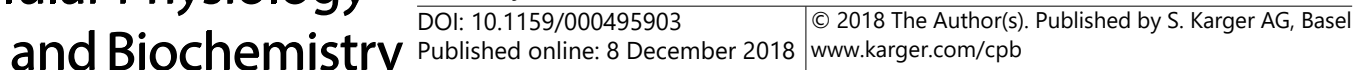 \\ Zhao et al.: CCL20 Promotes GCTB Progression}

\section{Cell culture}

GCTSCs and BMSCs were isolated from GCTB samples and bone marrow from normal donors as previously described $[3,6]$. RAW 264.7 cells were obtained from the Type Culture Collection of the Chinese Academy of Sciences. Cells were cultured in Minimum Essential Medium $\alpha$ ( $\alpha$-MEM; Gibco) supplemented with $10 \%$ fetal bovine serum (Hyclone) and $100 \mathrm{U} / \mathrm{ml}$ penicillin-streptomycin (Gibco). Cells were cultured in a humidified incubator (Thermo) with $5 \% \mathrm{CO}_{2}$ at $37^{\circ} \mathrm{C}$.

RNA extraction and gene expression analysis

Cells were collected and total RNA was extracted with TRIzol reagent (Invitrogen). Then, $500 \mathrm{ng}$ total RNA was reverse transcribed with PrimeScript ${ }^{\mathrm{TM}}$ RT Master Mix (Takara) according to the manufacturer's instructions. The complementary DNA was used for real-time PCR with SYBR Premix Ex Taq (Takara), and data analysis was performed using the $2^{-\Delta \Delta C t}$ method with normalization to the endogenous control $\beta$-actin. Primers are listed in Table 1.

\section{Enzyme-linked immunosorbent assay for serum CCL20}

An enzyme-linked immunosorbent assay (ELISA) was performed to determine the serum CCL20 protein levels. Briefly, 100- $\mu \mathrm{l}$ serum samples from each GCTB patient or normal donor were measured with a CCL20 ELISA kit (Invitrogen) according to the manufacturer's instructions.

Cell viability assay

The cell viability of GCTSCs was determined by the MTS method. After 48-h treatment with the indicated concentrations of CCL20, cells were counted with a VERSA Max Microplate Reader (Molecular Devices) as described previously [24].

\section{Transwell assay}

A Transwell assay was performed with Boyden chambers (Corning). GCTSCs or RAW264.7 cells were collected and re-suspended in blank $\alpha$-MEM after 12 -h serum starvation. In total, $6 \times 10^{4}$ cells were plated in the top chambers with or without the indicated concentrations of CCL20. The bottom chambers were filled with $600 \mu \mathrm{l}$ medium supplemented with $2 \%$ fetal bovine serum. After an 8 -h incubation, migrated cells were fixed with $4 \%$ paraformaldehyde and stained with $0.1 \%$ crystal violet. Bright-field images were obtained with an Olympus inverted microscope and migrated cells were counted using Image-Pro Plus 6.0.

\section{Mouse bone marrow monocyte isolation and osteoclast differentiation assay}

Mouse bone marrow monocytes (BMMs) were flushed and isolated from C57/BL6 mice as previously described [8]. Then, $8 \times 10^{3}$ BMMs were seeded into 96-well plates and incubated with $10 \mathrm{ng} / \mathrm{ml} \mathrm{M-CSF}, 50$ $\mathrm{ng} / \mathrm{ml}$ RANKL, and different concentrations of CCL20 or GCTSC-conditioned medium. After 5-7 days, the cells were fixed with $4 \%$ paraformaldehyde and permeabilized with $0.1 \%$ Triton-X 100 in phosphate-buffered saline for $5 \mathrm{~min}$. The cells were then subjected to tartrate-resistant acid phosphatase (TRAP) staining with a Leukocyte Acid Phosphatase kit (Sigma-Aldrich). TRAP-positive osteoclasts were photographed and then counted.

Table 1. Primer sequences used in this study

\begin{tabular}{lcc}
\hline Target gene & Forward primer $\left(5^{\prime}->3^{\prime}\right)$ & Reverse primer $\left(5^{\prime}->3^{\prime}\right)$ \\
\hline$\beta$-actin & GTACGCCAACACAGTGCTG & CGTCATACTCCTGCTTGCTG \\
Human CCL20 & TGCTGCTACTCCACCTCT & GCAAGTGAAACCTCCAAC \\
Human CCR6 & TGGCTGTTGGTTTGTGGA & AGGAGTAGGCAATCGGTA \\
Mouse Trap & GCTGGAAACCATGATCACCT & GAGTTGCCACACAGCATCAC \\
Mouse Nfatc1 & TGGAGAAGCAGAGCACAGAC & GCGGAAAGGTGGTATCTCAA \\
Mouse C-src & TCCTTTGGGATTCTGCTGAC & CCAGCACTGGCACATAAGG \\
Mouse DC-STAMP & GCTGTGGACTATCTGCTGTATCG & GACACGAGAATTTTAAGCTCTCCAC \\
Mouse Atp6v0d2 & GTTGCTATCCAGGACTCGGA & GTCATGTAGGTGAGAAATGT \\
\hline
\end{tabular}




\section{Cellular Physiology Cell Physiol Biochem 2018;51:2472-2483

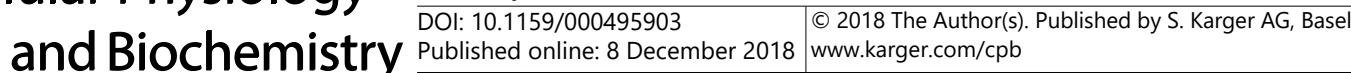 \\ Zhao et al.: CCL20 Promotes GCTB Progression}

Protein extraction and western blot analysis

Total protein was extracted using RIPA buffer. Samples were equally loaded in a $10 \%$ sodium dodecyl sulfate gel and transferred to nitrocellulose membranes. After being blocked with $5 \%$ bovine serum albumin, the membranes were incubated with the indicated primary antibodies overnight at $4^{\circ} \mathrm{C}$. Then, the membranes were incubated with DyLight 800-conjugated secondary antibody and scanned with LI-COR Infrared Imaged Odyssey (Gene Company Ltd.).

Luciferase reporter gene assay

Dual luciferase assays were conducted in a 24-well plate. Plasmids were transfected into $70 \%$ confluent HEK293 cells and RANKL (receptor activator for nuclear factor- $\kappa \mathrm{B}$ ligand) and different concentrations of rmCCL20 were added. After 48-h transfection, firefly and Renilla luciferase levels were quantified sequentially using the Dual Luciferase Assay kit (Promega) following the manufacturer's recommendations.

\section{Immunofluorescence staining}

RAW264.7 cells were seeded on glass coverslips and fixed with 4\% paraformaldehyde. After permeabilization and blocking with bovine serum albumin, the cells were incubated with primary antibody at $4^{\circ} \mathrm{C}$ overnight and secondary antibody for 2 -h at room temperature. The cells were then incubated with DAPI for 5-min and mounted on glass slides for microscope analysis.

\section{Statistical analysis}

The results are reported as mean \pm standard error of the mean (SEM). Significant differences between control and experimental groups were determined by an unpaired Student's t test. Survival curves were analyzed by a log-rank test. $p<0.05$ was considered significant.

\section{Results}

CCL20 is upregulated in GCTB patients and is a poor prognostic factor

Human microarray analysis of stromal cells was performed for five GCTB patients and five normal BMSCs (Fig. 1A). Bioinformatics analysis of chemokine family members revealed that CCL20 was significantly upregulated in GCTSCs with a fold change of 3.83 (Fig. 2B). To further confirm these findings, quantitative PCR $(10$ GCTSCs from patients versus 5 BMSCs) and RT-PCR (5 GCTSCs versus 5 BMSCs) were performed (Fig. 1C and D). The results indicated that CCL20 was significantly upregulated in GCTSCs. Meanwhile, the expression of CCR6, the receptor for CCL20, was also upregulated in GCTSCs.

Because CCL20 is a secretory protein, we next determined the serum CCL20 protein levels in 32 GCTB patients and 13 normal donors (Fig. 1E). The results indicated that CCL20 protein levels were dramatically upregulated in GCTB patients and were correlated with tumor size. Patients with larger tumors (diameters $>8 \mathrm{~cm}$ ) had higher levels of serum CCL20. A comparison of local recurrence and overall survival rates revealed that high levels of CCL20 indicated a worse prognosis (Fig. 1F). Finally, we performed receiver operating characteristic (ROC) curve analysis to determine the ability of CCL20 to predict GCTB prognosis (Fig. 1G). The area under the curve (AUC) was 0.8026 (95\% confidence interval, $0.6745-0.9313, p=0.002$ ), indicating good performance.

\section{CCL20 promotes GCTSC proliferation and migration in an autocrine manner}

Because CCR6 was upregulated in GCTSCs, we first investigated whether CCL20 acts as an autocrine factor. A Cell Counting Kit-8 cell viability assay revealed that recombinant human CCL20 (rhCCL20) dose-dependently upregulated GCTSC cell viability (Fig. 2A). Although classified as a benign tumor, GCTB can be associated with potentially aggressive behavior and a 1-9\% incidence of lung metastasis [9]. A Transwell assay indicated that rhCCL20 dramatically promoted GCTSC migration (Fig. 2B and C), which might at least partly explain the aggressiveness of GCTB. Both the proliferation and migration effects of CCL2 0 on GCTSCs were abolished by CCL20 antibody (Fig. 2D and E). However, neither of the effects was observed in BMSCs (Fig. $2 \mathrm{~F}$ and G). 
CCL20 recruits
BMMs to the tumor
microenvironment
and further promotes
osteoclastogenesis
The tumor microenvironment of GCTBs is considered to be osteolytic. It is widely accepted that the spindleshaped stromal cells can recruit mononuclear precursors to the tumor microenvironment and induce the formation of osteoclast-like giant cells. The expression was previously confirmed of CCR6 in BMMs [12]. Thus, in the current study, we examined the effects of CCL20 on BMMs. A Transwell assay showed that recombinant mouse CCL20 (rmCCL20) dosedependently promoted the migration of RAW264.7 cells (Fig. 3A and B). We separated the BMMs from 4-6-week-old mice and induced osteoclastogenesis with RANKL. The results indicated that CCL20 dosedependently promoted osteoclast differentiation (Fig. 3C). Statistical analysis revealed that both cell numbers (Fig. 3D for cells with $>5$ nuclei, Fig. 3E for cells with $>20$ nuclei) and areas of TRAP-positive cells (Fig. 3F) were increased after CCL20 treatment. According to a bone chip assay, bone erosion was exacerbated by CCL20 (Fig. $3 \mathrm{G}$ and $\mathrm{H}$ ).

The expression levels of osteoclast marker genes were further evaluated. Marker genes of osteoclast differentiation such as Trap, Nfatc1, and C-src (Fig. 4A-C) and cell fusion marker genes such as DC-STAMP and Atp6v0d2 (Fig. 4D and E) were all significantly elevated after CCL20 treatment.
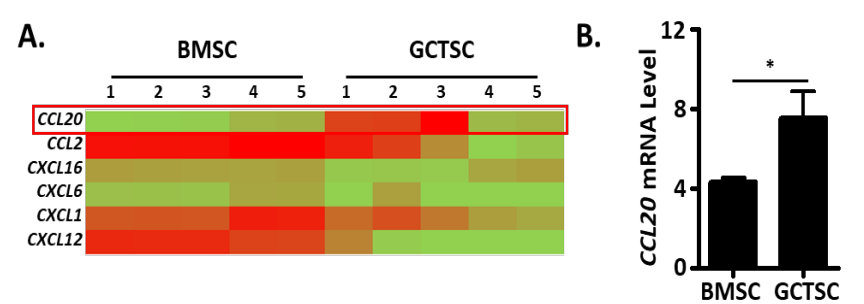

C.

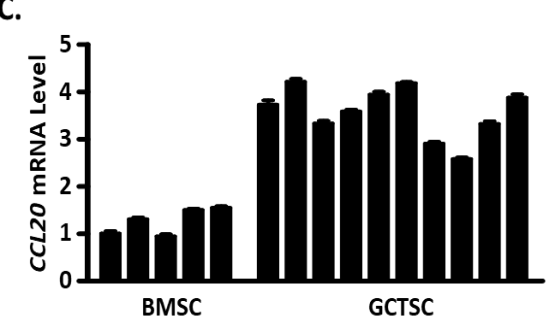

E.

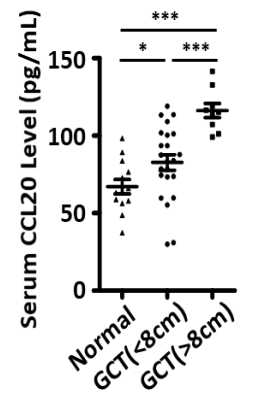

D.
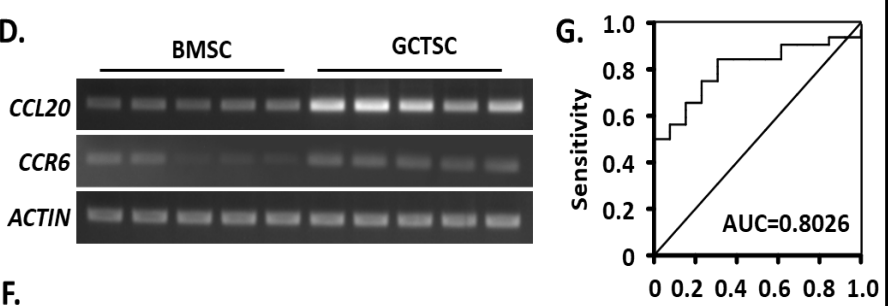

F.

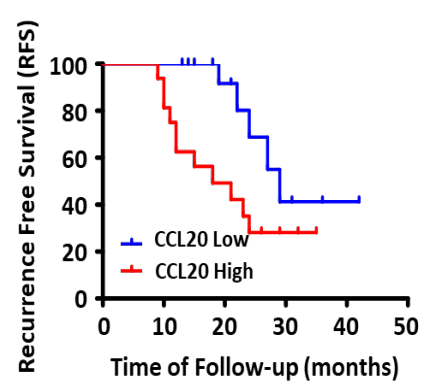

1-Specificity

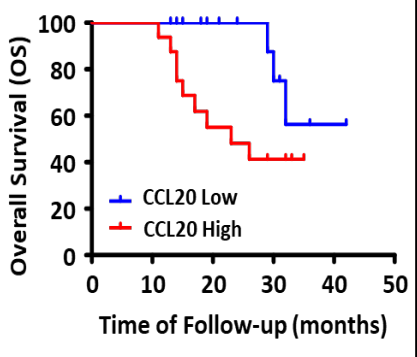

Fig. 1. CCL20 is upregulated in GCTB patients and is a poor prognostic factor. (A and B) RNA microarray data showing that CCL20 is upregulated in GCTSCs ( $\mathrm{n}=5$ ) compared with normal BMSCs $(\mathrm{n}=5)$. Error bars are mean \pm SEM. *, $p<0.05$. (C and D) Quantitative PCR and RT-PCR analyses of CCL20 mRNA levels in GCTB tumors ( $n=10 / 5)$ compared with normal stromal cells $(n=5)$. Error bars are mean \pm SEM. (E) ELISA of serum CCL20 in GCTB patients $\left(\mathrm{n}_{<8 \mathrm{~cm}}=23, \mathrm{n}_{>8 \mathrm{~cm}}=9\right)$ and normal donors $(\mathrm{n}=13)$. Error bars are mean \pm SEM. *, $\mathrm{p}<0.05$; ***, $\mathrm{p}<0.001$. (F) The serum CCL20 level was correlated with the local recurrence rate and overall survival of GCTB patients. $n=16$ for each group. Log-rank $\mathrm{p}<0.05$. (G) ROC curve for CCL20 in predicting GCTB. AUC $=0.8026$. 


\section{Overactive osteoclastogenesis is attenuated by CCL2O antibody}

Osteoclastogenesis can be induced by GCTSC-conditioned medium containing RANKL (Fig. 5A). Because CCL20 is secreted by GCTSCs, we used the monoclonal human CCL20 antibody to neutralize CCL20 in GCTSC-conditioned medium. Consistently, CCL20-exacerbated osteoclastogenesis was abolished by CCL20 antibody (Fig. 5A and $\mathrm{B})$. Osteoclast marker gene expression was also attenuated by CCL20 antibody treatment (Fig. 5C-G).

\section{CCL20 induces overactivation of the $A K T$ and $N F-\kappa B$ signaling pathways \\ To further reveal the} intrinsic mechanisms of CCL20 in GCTB progression, western blot analysis of the AKT and NF- $\kappa \mathrm{B}$ signaling pathways was performed (Fig. 6A). Phosphorylation of AKT, IкB $\alpha$, $\mathrm{IKK} \alpha / \beta$, and NF- $\kappa \mathrm{B}$ p65 were all enhanced after CCL20 treatment. Immunofluorescence showed that CCL20 promoted RANKLinduced NF- $\mathrm{KB}$ p65 nuclear transportation (Fig. 6B and C). A luciferase reporter gene assay revealed that the NF- $\kappa$ B luciferase activity of HEK293T cells was significantly upregulated after rhCCL20 treatment at the indicated concentrations (Fig. 6D). The AKT inhibitor MK2206 was used to further validate the activation of AKT signaling. CCL20-induced GCTSC proliferation and migration were all abolished by the addition of MK2206 (0.5 $\mu \mathrm{M})$ (Fig. 6E and F). All of these results indicated the overactivation of the AKT and NF- $\mathrm{kB}$ signaling pathways.

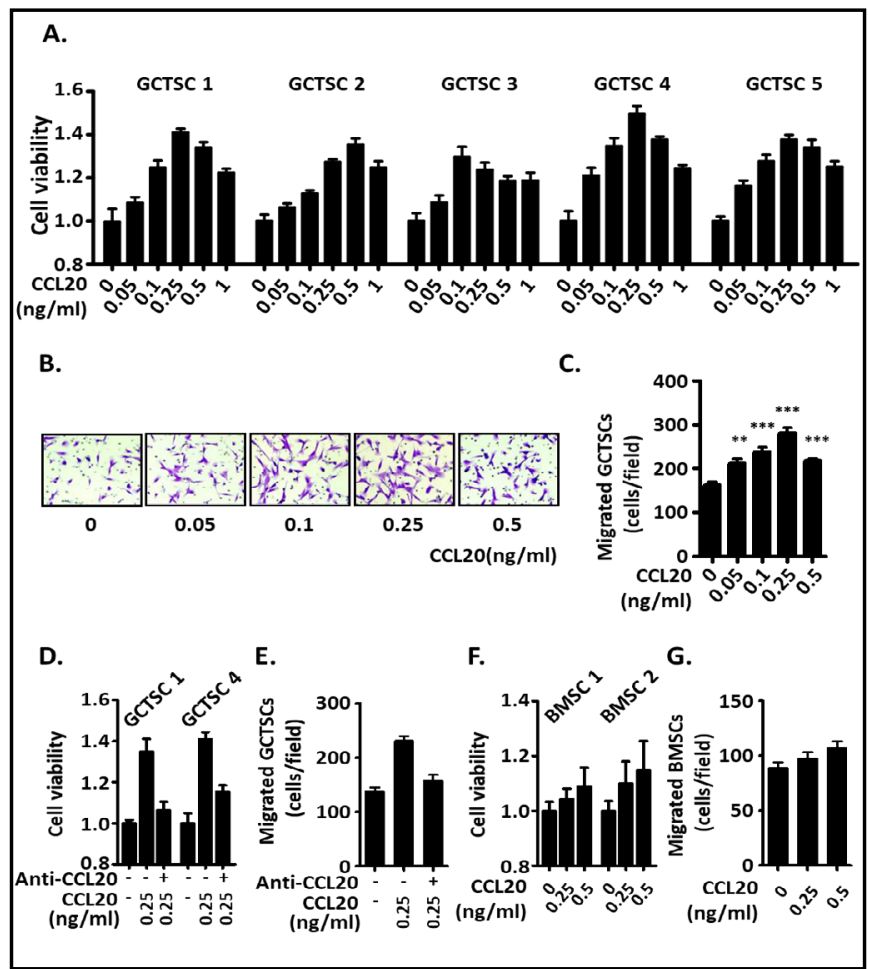

Fig. 2. CCL20 promotes GCTSC proliferation and migration in an autocrine manner. (A) Cell viability assay of GCTSCs from five patients after rhCCL20 treatment at the indicated concentrations. Error bars are mean \pm SEM of three independent experiments. (B and C) Transwell assay showing that rhCCL20 dramatically promotes GCTSC migration. Error bars are mean \pm SEM of three independent experiments. ${ }^{* *}, \mathrm{p}<0.01 ;{ }^{* * *}, \mathrm{p}<0.001$. (D and $\mathrm{E}$ ) CCL20-induced GCTSC proliferation and migration are abolished by human CCL20 antibody. Error bars are mean \pm SEM of three independent experiments. ( $F$ and G) CCL20 has little effect on BMSC proliferation and migration. Error bars are mean \pm SEM of three independent experiments. 
A.

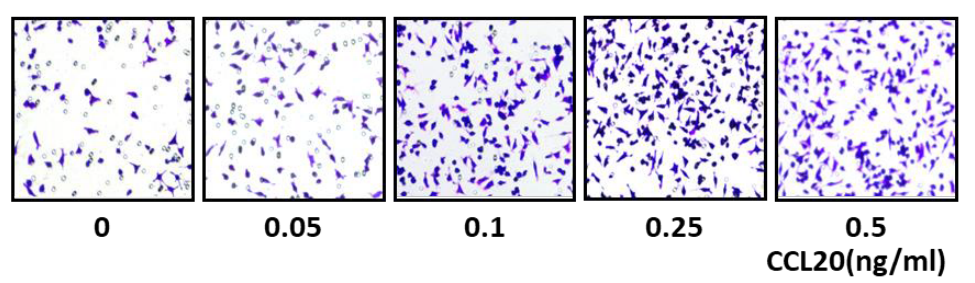

C.

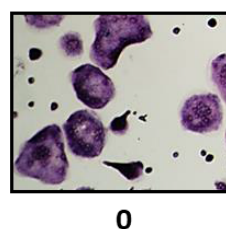

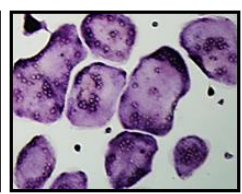

0.1

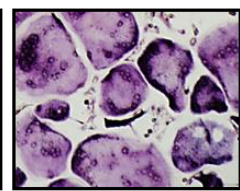

0.25

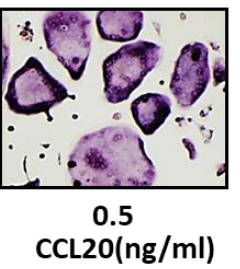

B.

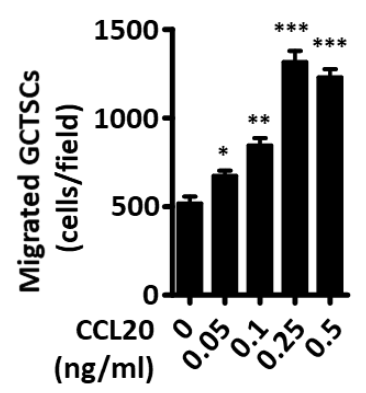

D.

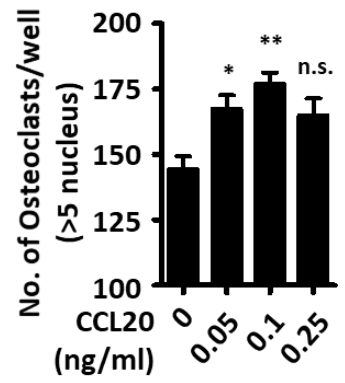

E.

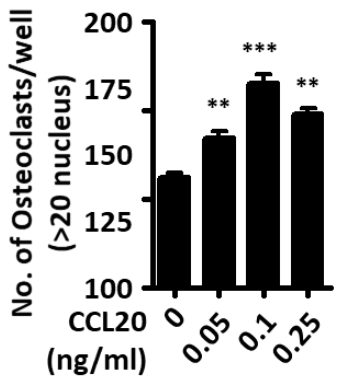

F.

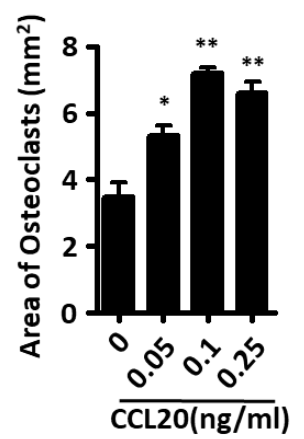

G.

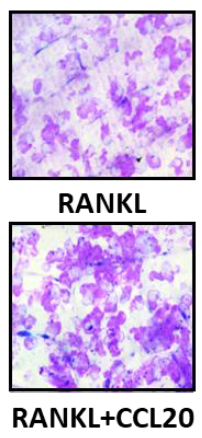

H.

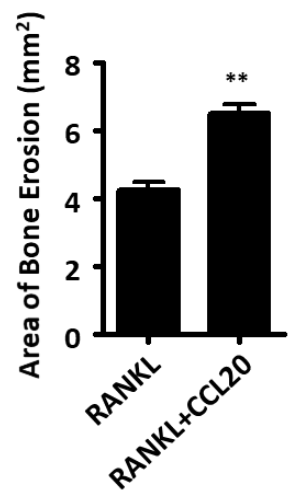

Fig. 3. CCL20 recruits BMMs to the tumor microenvironment and further promotes osteoclastogenesis. (A and B) Transwell assay showing that rmCCL20 can dose-dependently promote RAW264.7 cell migration. Error bars are mean \pm SEM of three independent experiments. ${ }^{*}, \mathrm{p}<0.05 ;{ }^{* *}, \mathrm{p}<0.01 ;{ }^{* *}, \mathrm{p}<0.001$. (C-F) RANKL-induced osteoclastogenesis is dose-dependently promoted by CCL20 treatment and leads to larger osteoclasts. Error bars are mean \pm SEM of three independent experiments. n.s., not significant; *, p<0.05; ${ }^{* *}$, $\mathrm{p}<0.01$; ${ }^{* * *}, \mathrm{p}<0.001$. ( $\mathrm{G}$ and $\mathrm{H}$ ) Bone chip assay showing the aggressiveness of bone erosion after rmCCL20 treatment. Error bars are mean \pm SEM of three independent experiments. ${ }^{* *}, \mathrm{p}<0.01$. 
Fig. 4. Osteoclast marker genes are significantly upregulated after CCL20 treatment. Error bars are mean \pm SEM of three independent experiments.

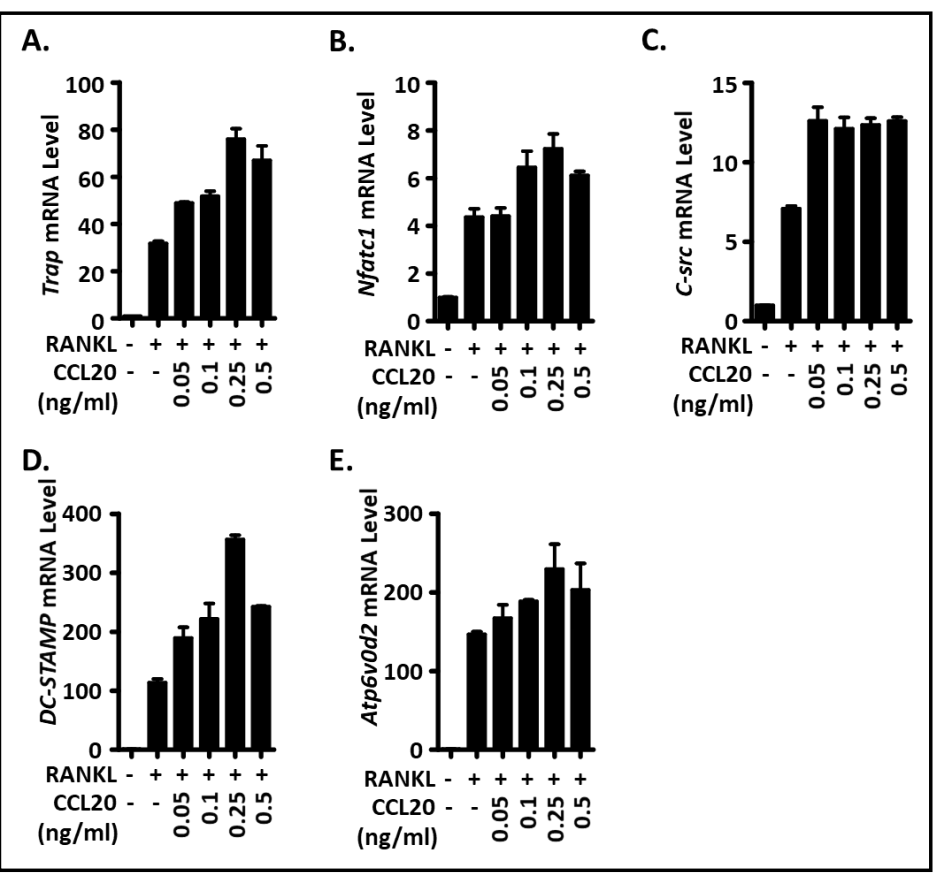

Fig. 5. Overactive osteoclastogenesis can be attenuated by CCL20 antibody. (A andB) GCTSC-conditionedmediuminduced osteoclastogenesis is significantly inhibited by human CCL20 antibody. Error bars are mean \pm SEM of three independent experiments. ${ }^{* *}, \mathrm{p}<0.01$. (C-G) The upregulation of osteoclast marker genes is attenuated by CCL20 antibody treatment. Error bars are mean \pm SEM of three independent experiments.

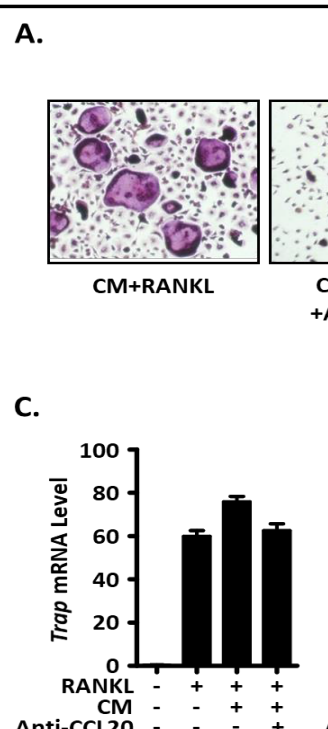

CM
Anti-CCL20

F.

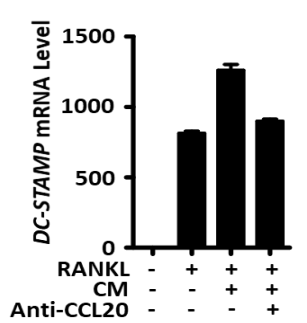

D.

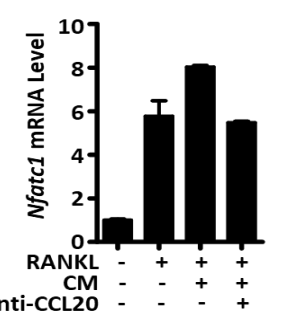

E.

G.

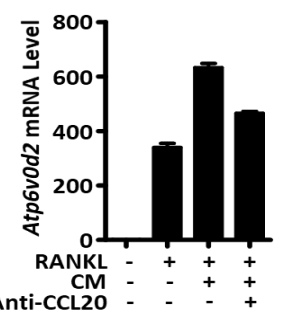




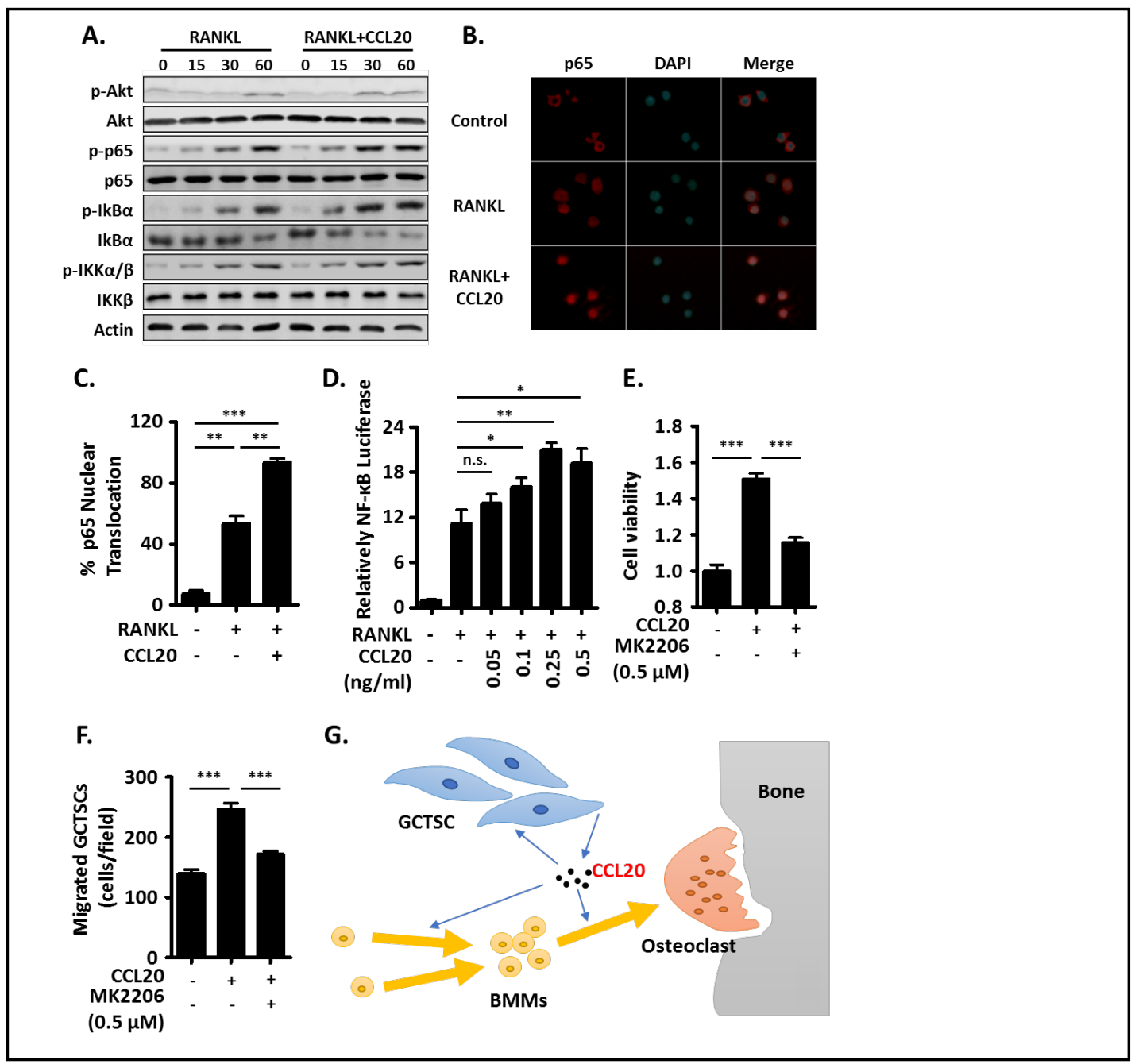

Fig. 6. CCL20 induces the overactivation of the AKT and NF- $\kappa B$ signaling pathways. (A) Western blot showing enhanced phosphorylation of the AKT and NF- $\kappa B$ signaling pathways after CCL20 treatment. (B and C) Immunofluorescence showing that CCL20 promotes RANKL-induced NF- $\kappa$ B p65 nuclear transportation. Error bars are mean \pm SEM of three independent experiments. ${ }^{* *}, \mathrm{p}<0.01$; ${ }^{* *}, \mathrm{p}<0.001$. (D) Relative NF- $\kappa \mathrm{B}$ luciferase activity of HEK293T cells after rmCCL20 treatment at the indicated concentrations. Error bars are mean \pm SEM of three independent experiments. n.s., not significant; ${ }^{* *}, \mathrm{p}<0.01 ;{ }^{* *}, \mathrm{p}<0.001$. (E and F) CCL20-induced GCTSC proliferation and migration are abolished by the AKT inhibitor MK2206. Error bars are mean \pm SEM of three independent experiments. n.s., not significant; ${ }^{* * *}, \mathrm{p}<0.001$. (G) CCL20 is secreted by GCTSCs and acts as a key modulator in the pathological progression of GCTB.

\section{Discussion}

Although GCTBs are predominantly considered benign and to show an osteolytic local aggressive lesion, some GCTBs show unpredictable behaviors. The term "malignancy in GCTB" is used by the World Health Organization to describe such tumors, which show malignant potential and can completely transform into malignant tumors [25, 26]. Currently, preoperative prediction and postoperative monitoring of GCTB patient prognosis are limited and mostly rely on patients' general information, radiological features, treatment history, and tumor pathological reports $[1,4,5]$. Therefore, identification of sensitive and specific factors that can predict tumor progression and prognosis, particularly those found in the serum, are urgently required. In the present study, we found that CCL20 was upregulated in both tumor samples and patient serum and could be used as an independent prognostic 


\section{Cellular Physiology Cell Physiol Biochem 2018;51:2472-2483 \begin{tabular}{ll|l} 
and Biochemistry & $\begin{array}{l}\text { DOl: 10.1159/000495903 } \\
\text { Publisned online: 8 December } 2018\end{array}$ & $\begin{array}{l}\text { (2018 The Author(s). Published by S. Karger AG, Basel } \\
\text { www.karger.com/cpb }\end{array}$ \\
\cline { 2 - 3 }
\end{tabular} \\ Zhao et al.: CCL20 Promotes GCTB Progression}

factor with good performance $(\mathrm{AUC}=0.8026)$ that was correlated with tumor progression.

CCL20 has been identified in several solid tumors and proven to play important roles in tumor progression [27]. However, the function of CCL20 in GCTB remains elusive. GCTSCs are currently accepted as the tumor component of GCTB and can recruit mononuclear cells to form giant cells. These giant cells can further absorb bone matrix and lead to bone breakdown $[3,6,7]$. RANKL/RANK are widely accepted to be key regulators of bone physiology that control osteoclast development. In the present study, we revealed the upregulation of CCL20 in GCTSCs and identified its functions in two respects. We found that CCL20 could act as an autocrine cytokine to promote GCTSC proliferation and migration, which might contribute to the malignant performance of GCTB. Meanwhile, CCL20 could also recruit mononuclear cells and promote RANKL-induced osteoclastogenesis in a paracrine manner, which may reflect the osteolytic clinical features of GCTB.

The treatment strategies for GCTB are limited to surgical resection $[8,28]$, although bisphosphonates are effective in the local control of GCTB-induced osteolysis $[1,29,30]$. The monoclonal human RANKL antibody denosumab has been approved by the American Food and Drug Administration for unresectable GCTB [31]. Clinical experiences indicated that, after denosumab treatment, GCTB tumors are under control and that parts of unresectable tumors become resectable. The typically red and soft tumors become gray and hard, and pathological examination identifies few giant cells, but abundant GCTSCs, after denosumab treatment [32, 33]. However, both bisphosphonates and denosumab show limitations in the control of GCTSC progression. In this study, we demonstrated the dual signaling functions of CCL20 and indicated the potential therapeutic value of CCL20 targeting in GCTB patients.

Our previous studies and the literature have shown that the NF- $\kappa B$ signaling pathway, which plays an important role in tumor progression and osteoclast differentiation, is overactive in GCTB $[3,8,34]$. In addition, CCL2 0 is reported to promote the phosphorylation of AKT [35]. Overactivation of AKT signaling can also induce the upregulation of NFATC1 and further promote osteoclastogenesis [36]. At the same time, overactivation of AKT can also promote tumor proliferation and migration [35, 37, 38]. Our results indicated that GCTSC-secreted CCL20 could significantly induce the overactivation of both the AKT and NF- $\mathrm{KB}$ pathways during osteoclast differentiation. Additionally, the upregulation of osteoclastogenesis marker genes was abolished with CCL20 antibody treatment. All of these clues indicated that CCL20 can promote GCTB tumor progression, at least partly by regulating the AKT and NF- $\mathrm{BB}$ pathways.

\section{Conclusion}

Taken together, our data suggest that GCTSC-secreted CCL20 acts as a key modulator of the pathological progression of GCTB. It can promote GCTSC proliferation and migration in an autocrine manner and can recruit BMMs to the tumor microenvironment and enhance osteoclastogenesis in a paracrine manner (Fig. 6G). These findings strongly indicate the potential prognostic and therapeutic value of CCL20 in GCTB.

\section{Acknowledgements}

This work was supported by grants from the National Key Research and Development Program of China (2016YFC0902100), National Natural Science Foundation of China (81572640; 81330059), and the Science and Technology Commission of Shanghai Municipality (17ZR143000). 


\section{Cellular Physiology Cell Physiol Biochem 2018;51:2472-2483 \begin{tabular}{l|l|l} 
and Biochemistry Published onlIne: 8 December 2018 & $\begin{array}{l}\text { (c) } 2018 \text { The Author(s). Published by S. Karger AG, Basel } \\
\text { www.karger.com/cpb }\end{array}$ \\
\hline
\end{tabular} \\ Zhao et al.: CCL20 Promotes GCTB Progression}

\section{Disclosure Statement}

The authors have no conflicts of interest to declare.

\section{References}

1 Xu W, Li X, Huang W, Wang Y, Han S, Chen S, Xu L, Yang X, Liu T, Xiao J: Factors affecting prognosis of patients with giant cell tumors of the mobile spine: retrospective analysis of 102 patients in a single center. Ann Surg Oncol 2013;20:804-810.

-2 Amanatullah DF, Clark TR, Lopez MJ, Borys D, Tamurian RM: Giant cell tumor of bone. Orthopedics 2014;37:112-120.

-3 Li B, Qian M, Cao H, Jia Q, Wu Z, Yang X, Ma T, Wei H, Chen T, Xiao J: TGF- $\beta 2$-induced ANGPTL4 expression promotes tumor progression and osteoclast differentiation in giant cell tumor of bone. Oncotarget 2017;8:54966-54977.

4 Cheng DD, Hu T, Zhang HZ, Huang J, Yang QC: Factors Affecting the Recurrence of Giant Cell Tumor of Bone After Surgery: A Clinicopathological Study of 80 Cases from a Single Center. Cell Physiol Biochem 2015;36:1961-1970.

5 Li J, Li B, Zhou P, Zhao J, Wu Z, Yang X, Wei H, Chen T, Xiao J: Nomograms for prognostic factors of spinal giant cell tumor combining traditional clinical characteristics with inflammatory biomarkers after gross total resection. Oncotarget 2017;8:86934-86946.

-6 Song D, Meng T, Xu W, Hou T, Lin Z, Yin H, Li B, Zhou L, Wang T, Han S, Fan T, Miao W, Liu M, Luo J, Zhou W, Li Z, Xiao J: 5-Fluoruracil blocked giant cell tumor progression by suppressing osteoclastogenesis through NF-kappaB signals and blocking angiogenesis. Bone 2015;78:46-54.

-7 Xu M, Song ZG, Xu CX, Rong GH, Fan KX, Chen JY, Zhang W, Jia JP, Han G, Wang W, Chai W, Liang WT, Bi WZ, Wang Y: IL-17A stimulates the progression of giant cell tumors of bone. Clin Cancer Res 2013;19:46974705 .

8 Xu L, Luo J, Jin R, Yue Z, Sun P, Yang Z, Yang X, Wan W, Zhang J, Li S, Liu M, Xiao J: Bortezomib Inhibits Giant Cell Tumor of Bone through Induction of Cell Apoptosis and Inhibition of Osteoclast Recruitment, Giant Cell Formation, and Bone Resorption. Mol Cancer Ther 2016;15:854-865.

99 Yang Y, Huang Z, Niu X, Xu H, Li Y, Liu W: Clinical characteristics and risk factors analysis of lung metastasis of benign giant cell tumor of bone. J Bone Oncol 2017;7:23-28.

10 Allavena P, Germano G, Marchesi F, Mantovani A: Chemokines in cancer related inflammation. Exp Cell Res 2011;317:664-673.

11 Lancaster JN, Li Y, LIR E: Chemokine-Mediated Choreography of Thymocyte Development and Selection. Trends Immunol 2018;39:86-98.

12 Giuliani N, Lisignoli G, Colla S, Lazzaretti M, Storti P, Mancini C, Bonomini S, Manferdini C, Codeluppi K, Facchini A, Rizzoli V: CC-chemokine ligand 20/macrophage inflammatory protein-3 $\alpha$ and CC-chemokine receptor 6 are overexpressed in myeloma microenvironment related to osteolytic bone lesions. Cancer Res 2008;68:6840-6850.

13 Zlotnik A, Yoshie 0: The chemokine superfamily revisited. Immunity 2012;36:705-716.

-14 Mukaida N, Baba T: Chemokines in tumor development and progression. Exp Cell Res 2012;318:95-102.

15 Gál P, Varinská L, Fáber L, Novák Š, Szabo P, Mitrengová P, Mirossay A, Mučaji P, Smetana K: How Signaling Molecules Regulate Tumor Microenvironment: Parallels to Wound Repair. Molecules 2017;22.

16 Lee AY, Körner H: CCR6/CCL20 chemokine axis in human immunodeficiency virus immunity and pathogenesis. J Gen Virol 2017;98:338-344.

17 Zhao L, Xia J, Wang X, Xu F: Transcriptional regulation of CCL20 expression. Microbes Infect 2014;16:864870 .

18 Lee AY, Körner H: CCR6 and CCL20: emerging players in the pathogenesis of rheumatoid arthritis. Immunol Cell Biol 2014;92:354-358.

19 Frick VO, Rubie C, Keilholz U, Ghadjar P: Chemokine/chemokine receptor pair CCL20/CCR6 in human colorectal malignancy: An overview. World J Gastroenterol 2016;22:833-841.

-20 Muscella A, Vetrugno C, Marsigliante S: CCL20 promotes migration and invasiveness of human cancerous breast epithelial cells in primary culture. Mol Carcinog 2017;56:2461-2473. 


\section{Cellular Physiology Cell Physiol Biochem 2018;51:2472-2483

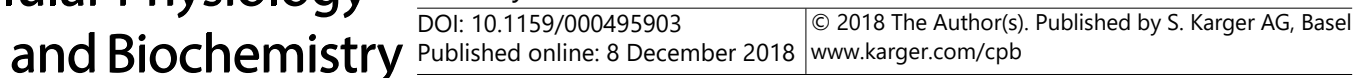 \\ Zhao et al.: CCL20 Promotes GCTB Progression}

21 Lee SK, Park KK, Kim HJ, Park J, Son SH, Kim KR, Chung WY: Human antigen R-regulated CCL20 contributes to osteolytic breast cancer bone metastasis. Sci Rep 2017;7:9610.

22 Liu B, Jia Y, Ma J, Wu S, Jiang H, Cao Y, Sun X, Yin X, Yan S, Shang M, Mao A: Tumor-associated macrophagederived CCL20 enhances the growth and metastasis of pancreatic cancer. Acta Biochim Biophys Sin (Shanghai) 2016;48:1067-1074.

23 Du D, Liu Y, Qian H, Zhang B, Tang X, Zhang T, Liu W: The effects of the CCR6/CCL20 biological axis on the invasion and metastasis of hepatocellular carcinoma. Int J Mol Sci 2014;15:6441-6452.

-24 Li Z, Xiao J, Wu X, Li W, Yang Z, Xie J, Xu L, Cai X, Lin Z, Guo W, Luo J, Liu M: Plumbagin inhibits breast tumor bone metastasis and osteolysis by modulating the tumor-bone microenvironment. Curr Mol Med 2012;12:967-981.

25 Bertoni F, Bacchini P, Staals EL: Malignancy in giant cell tumor of bone. Cancer 2003;97:2520-2529.

-26 Yin H, Cheng M, Li B, Li B, Wang P, Meng T, Wang J, Zhou W, Yan W, Xiao J: Treatment and outcome of malignant giant cell tumor in the spine. J Neurooncol 2015;124:275-281.

27 Ben-Baruch A: The multifaceted roles of chemokines in malignancy. Cancer Metastasis Rev 2006;25:357371.

28 van der Heijden L, Dijkstra PD, van de Sande MA, Kroep JR, Nout RA, van Rijswijk CS, Bovée JV, Hogendoorn PC, Gelderblom H: The clinical approach toward giant cell tumor of bone. Oncologist 2014;19:550-561.

-29 Cornelis F, Truchetet ME, Amoretti N, Verdier D, Fournier C, Pillet O, Gille O, Hauger O: Bisphosphonate therapy for unresectable symptomatic benign bone tumors: a long-term prospective study of tolerance and efficacy. Bone 2014;58:11-16.

-30 Tse LF, Wong KC, Kumta SM, Huang L, Chow TC, Griffith JF: Bisphosphonates reduce local recurrence in extremity giant cell tumor of bone: a case-control study. Bone 2008;42:68-73.

-31 Lewin J, Thomas D: Denosumab: a new treatment option for giant cell tumor of bone. Drugs Today (Barc) 2013;49:693-700.

32 Branstetter DG, Nelson SD, Manivel JC, Blay JY, Chawla S, Thomas DM, Jun S, Jacobs I: Denosumab induces tumor reduction and bone formation in patients with giant-cell tumor of bone. Clin Cancer Res 2012;18:4415-4424.

33 Wendling D, Guillot X, Sondag M, Chaigneau L, Aubry S, Prati C: Giant cell tumor of bone under Denosumab treatment. Joint Bone Spine 2018;85:491.

-34 de Castro Barbosa ML, da CRA, AGM F, Camarinha BD, de Carvalho Silva GC, AGF L, Cardoso EA, de Oliveira Freitas Lione V: NF- $\kappa B$ Signaling Pathway Inhibitors as Anticancer Drug Candidates. Anticancer Agents Med Chem 2017;17:483-490.

35 Marsigliante S, Vetrugno C, Muscella A: CCL20 induces migration and proliferation on breast epithelial cells. J Cell Physiol 2013;228:1873-1883.

-36 Wu M, Chen W, Lu Y, Zhu G, Hao L, Li YP: Ga13 negatively controls osteoclastogenesis through inhibition of the Akt-GSK3ß-NFATc1 signalling pathway. Nat Commun 2017;8:13700.

-37 Hou T, Zhou L, Wang L, Kazobinka G, Zhang X, Chen Z: CLCA4 inhibits bladder cancer cell proliferation, migration, and invasion by suppressing the PI3K/AKT pathway. Oncotarget 2017;8:93001-93013.

>38 Ooms LM, Binge LC, Davies EM, Rahman P, Conway JR, Gurung R, Ferguson DT, Papa A, Fedele CG, Vieusseux JL, Chai RC, Koentgen F, Price JT, Tiganis T, Timpson P, McLean CA, Mitchell CA: The Inositol Polyphosphate 5-Phosphatase PIPP Regulates AKT1-Dependent Breast Cancer Growth and Metastasis. Cancer Cell 2015;28:155-169. 